\title{
A case of a de-novo lesion in the left circumflex artery treated with excimer laser and drug-coated balloon under the guidance of optical frequency domain imaging
}

\author{
Shinichiro Masuda ${ }^{1}$, Takashi Shibui ${ }^{1}$, Takaaki Tsuchiyama ${ }^{1}$, Takashi Ashikaga ${ }^{2}$ \\ ${ }^{1}$ Department of Cardiology, Tokyo Metropolitan Hiroo Hospital, Tokyo, Japan \\ ${ }^{2}$ Department of Cardiology, Japanese Red Cross Musashino Hospital, Tokyo, Japan
}

A 72-year-old man who previously underwent percutaneous coronary intervention with a drugeluting stent implantation from the left main trunk and extending to proximal left anterior descending artery was admitted to the documented hospital for angina pectoris. Coronary angiography (CAG) revealed $90 \%$ stenosis at the ostium of the left circumflex artery (LCX) (Fig. 1A). Excimer laser coronary angioplasty (ELCA) was performed using a $0.9 \mathrm{~mm}$ concentric laser catheter at a pulse rate of $25 \mathrm{~Hz}$ and energy output of $45 \mathrm{~mJ} / \mathrm{mm}^{2}, 35 \mathrm{~Hz}$ and $55 \mathrm{~mJ} / \mathrm{mm}^{2}$, and $45 \mathrm{~Hz}$ and $60 \mathrm{~mJ} / \mathrm{mm}^{2}$ for a total of 5200 pulses and balloon angioplasty using a drugcoated balloon (DCB) under the guidance of optical frequency domain imaging (OFDI), which revealed fibrous plaque and eccentric severe calcification (Fig. 1B). After ELCA, minimum lumen area (MLA) increased from $1.4 \mathrm{~mm}^{2}$ to $2.6 \mathrm{~mm}^{2}$ (Fig. 1C) and on final OFDI to $3.9 \mathrm{~mm}^{2}$ along with minor plaque dissection (Fig. 1D). Final CAG demonstrated optimal result without flow limitation (Fig 1E). After discharge, no significant clinical events were reported. Eight months later, follow-up CAG and OFDI were performed. Follow-up CAG demonstrated no restenosis at the ostium of the LCX (Fig. 1F). OFDI showed that the MLA slightly decreased from $3.9 \mathrm{~mm}^{2}$ to $3.5 \mathrm{~mm}^{2}$ and that the minor dissection had clearly improved (Fig. 1G). The DCB is efficacious in de-novo coronary artery lesions [1], which mainly contributed to suppress the restenosis in this case; however, although OFDI after ELCA demonstrated a slight increase in MLA, ELCA might be attributed to the lesion debulking and modification leading to optimal balloon expansion. A similar mechanism was previouslyreportedin the case ofin-stentrestenosis [2]. For acute myocardial infarction, the combined use of ELCA and DCB for de-novo coronary artery disease works synergistically to reduce restenosis [3]. Stent-less strategy employing ELCA and DCB may be an effective revascularization of large vessel denovo lesions, when traditional stent deployment is not a viable option.

Informed consent was obtained from the patient in accordance with the Helsinki Declaration.

\section{Acknowledgements}

The authors wish to thank Dr. Richard H. Kaszynski for reviewing and revising this manuscript.

Conflict of interest: None declared

\section{References}

1. Uskela S, Kärkkäinen JM, Eränen J, et al. Percutaneous coronary intervention with drug-coated balloon-only strategy in stable coronary artery disease and in acute coronary syndromes: An allcomers registry study. Catheter Cardiovasc Interv. 2019; 93(5): 893-900, doi: 10.1002/ccd.27950, indexed in Pubmed: 30380186.

2. Yin Da, Maehara A, Mezzafonte S, et al. Excimer laser angioplasty-facilitated fracturing of napkin-ring peri-stent calcium in a chronically underexpanded stent: documentation by optical coherence tomography. JACC Cardiovasc Interv. 2015; 8(8): e137-e139, doi: 10.1016/j.jcin.2015.02.018, indexed in Pubmed: 26205458.

3. Harima A, Sairaku A, Inoue I, et al. Real-life experience of a stent-less revascularization strategy using a combination of excimer laser and drug-coated balloon for patients with acute coronary syndrome. J Interv Cardiol. 2018; 31(3): 284-292, doi: 10.1111/joic.12495, indexed in Pubmed: 29464846.

Address for correspondence: Shinichiro Masuda, MD, Department of Cardiology, Tokyo Metropolitan Hiroo Hospital, 2-34-10, Ebisu, Shibuya-ku, Tokyo, 150-0013, Japan, tel: +08-3-3444-1181, fax: +08-3-3444-3196,

e-mail: giga627@yahoo.co.jp

Received: 24.07.2018 Accepted: 28.01.2019 




Figure 1. Coronary angiography (CAG) and optical frequency domain imaging (OFDI) findings of the culprit lesion at the time of percutaneous coronary intervention (PCI) and subsequent follow-up CAG. A. CAG before PCl showing severe stenosis at the ostium of the left circumflex artery (white arrow); B. OFDI image before PCI showing eccentric severe calcification (asterisks), and a minimum lumen area (MLA) of $1.4 \mathrm{~mm}^{2}$; C. OFDI image after excimer laser coronary angioplasty with MLA of $2.6 \mathrm{~mm}^{2}$; D. Final OFDI image at the PCl demonstrates a small plaque dissection (white arrows) and MLA of $3.9 \mathrm{~mm}^{2}$; E. CAG after PCl showing optimal results; F. CAG at follow-up shows no restenosis; G. OFDI at follow-up CAG showing improvements in plaque dissection and a slight reduction in the MLA from $3.9 \mathrm{~mm}^{2}$ to $3.6 \mathrm{~mm}^{2}$. 